\title{
Value of 18F-fluorodeoxyglucose positron emission tomography/computed tomography (FDG PET/CT) in assessment of response to preoperative chemotherapy in pediatric sarcoma
}

\author{
Ahmed Mohamed Rashad ${ }^{1}$, Ahmed Mohamed Abougabal ${ }^{1 *}$, Shady Hassan Fadel${ }^{2}$, Walid Mohamed Omar ${ }^{3}$ and \\ Khaled Mohamed Moghazy
}

\begin{abstract}
Background: The aim of this retrospective study was to highlight the role of FDG PET/CT in the assessment of tumor response to preoperative chemotherapy in pediatric sarcoma. Eighteen patients were included in our study: 13 patients were males and 5 were females ranging between 1 and 18 years with a mean age of 13.3 years. The patients had pathologically proven osseous or soft tissue sarcoma. All patients underwent sequential 18F-FDG PET/ CT before (PET-CT1) and after (PET-CT2) neoadjuvant chemotherapy. Maximum standardized uptake value (SUVmax) was measured in the primary lesion on PET/CT1 (SUV1) and PET/CT2 (SUV2). After surgery, the effects of neoadjuvant chemotherapy were evaluated histopathologically: $\geq 90 \%$ necrosis indicated was considered a good response and $<90 \%$ necrosis was considered a poor response. The correlation between SUV2 and the histologic response was assessed.
\end{abstract}

Results: The sensitivity, specificity, positive predictive value, and negative predictive value of SUV2 for assessment of treatment response were 100\%, 91.67\%, 85.71\%, and 100\%, respectively. The overall accuracy was found to be $98.3 \%$.

Conclusions: $18 \mathrm{~F}-\mathrm{FDG}$ PET/CT provides a reliable non-invasive diagnostic tool in assessment of response to preoperative chemotherapy in pediatric sarcoma.

Keywords: PET/CT, Sarcoma, Chemotherapy

\section{Background}

Pediatric sarcomas differ according to histologic subtype, but as a whole, represent about $13 \%$ of all pediatric malignancies [1].

The most common are soft tissue sarcomas. Soft tissue sarcomas have wide range according to genetic makeup, have more than 50 histologic subtypes, and are often associated with differing clinical and prognostic features [2]. These tumors, which arise in the soft tissues namely muscle, fat, blood vessels, nerves, tendons, or synovium,

\footnotetext{
* Correspondence: abougabal74@yahoo.com

'Diagnostic and Interventional Radiology Department, Faculty of Medicine,

Alexandria University, Alexandria, Egypt

Full list of author information is available at the end of the article
}

include embryonal rhabdomyosarcoma in children, synovial sarcoma in young adults, and high-grade pleomorphic sarcoma, liposarcoma, and leiomyosarcoma in adults. The extremities (most common in the thigh) are the most common location. Few otherwise can occur in the chest wall and retroperitoneum [1].

On the other hand, the most common primary malignant bone tumors in pediatric age are osteosarcoma and Ewing sarcoma [3]. Osteosarcoma originates from primitive bone-forming mesenchymal stem cells and mainly occurs in the metaphyseal portion of the long bones [4]. Ewing sarcoma equally originates from the axial and the appendicular skeleton [5]. 
Standard preoperative treatment of bone sarcomas and some subtypes or stages of soft tissue sarcoma includes chemotherapy. A timely noninvasive evaluation of response to chemotherapy is recommended to improve patient management and to avoid unnecessary toxicity and costs. Sarcomas can also be adequately controlled with surgical excision and adjuvant radiotherapy [6].

Anatomic imaging alone is not accurate enough for evaluation of bone and soft tissue tumors after the end of treatment, the sequel to posttreatment changes, including dysmorphic normal anatomy, disruption of normal tissue planes, and metal artifacts [7].

Evaluation of response by size-based criteria as defined in the response evaluation criteria in solid tumors (RECIST) criteria has its limitations and is of questionable value in the response evaluation of sarcomas [8].

Sarcomas are histologically heterogeneous tumors that vary in growth rate and contain a large volume of nonmalignant cells or extracellular material. For example, in slowly growing sarcomas, a slow progression during chemotherapy may be misinterpreted as a stable disease and thus a response to chemotherapy by $\mathrm{CT}$, as the tumor volume has hardly changed in the interval between two studies. The volumes of nonmalignant material in the tumor, or the replacement of malignant parts by fibrous material, can form the rest mass after chemotherapy. Therefore, the response to chemotherapy does not necessarily include a reduction of the size of the tumor $[9,10]$.

Percentage necrosis is prognostic in bone sarcomas, in Ewing sarcoma, 5-year disease-free survival (DFS) was $95 \%$ in good responders whereas $34 \%$ in poor responders [11]. After neoadjuvant chemotherapy for osteosarcoma, patients with $<10 \%$ viable tumor or $\geq 90 \%$ tumor necrosis at the time of surgical excision are classified as "good responders" [12].

Furthermore, a high SUVmax following neoadjuvant chemotherapy commonly associated with a worse prognosis [13], whereas low SUVmax after neoadjuvant chemotherapy was shown to correlate with an improved progression-free survival (PFS) [14].

It was also suggested that complete metabolic response (CMR) in patients with rhabdomyosarcoma following neoadjuvant chemotherapy and radiation therapy is associated with an improved local relapse-free survival [15].

The histologic response following neoadjuvant chemotherapy was used by several investigators to guide the selection of alternative postoperative chemotherapy in an attempt to improve event-free survival (EFS) [16].

Fluorodeoxyglucose (FDG)-PET/computed tomography (CT) is an imaging modality, which includes both anatomic localization by $\mathrm{CT}$ and functional characterization using PET imaging and which has an emerging role in the assessment of sarcomas [17].
And so, FDG-PET/CT imaging is a noninvasive modality for restaging and assessment of treatment response of patients after sarcoma treatment and has been found to have a profound effect on overall prognosis [18].

The aim of this retrospective study was to highlight the role of FDG PET/CT in the assessment of therapy response in pediatric sarcoma.

\section{Material and methods}

Eighteen patients in the pediatric age group were included in this retrospective study. The patients had pathologically proven osseous or soft tissue sarcoma, who were evaluated for degree of response to chemotherapy in the immediate preoperative period. None of our cases underwent surgery before completion of therapy. All the patients were subjected to thorough history taking, clinical examination, and F18-FDG PET/CT.

F18-FDG PET/CT study was done twice, one before administration of chemotherapy (SUV1) and one after finishing chemotherapy just before surgery (SUV2) using a dedicated PET/CT scanner (Biograph, True-Point; Siemens). The study was done about 4-6 weeks after the last cycle of chemotherapy. Fortunately, all the examined cases were nondiabetics, so there was no need for specific radiotracer dose adjustment.

This machine integrated a PET scanner with a dualsection helical CT scanner (40 slice Emotion; Siemens), and this allows the acquisition of co-registered $\mathrm{CT}$ and PET images in one session.

The CT protocol included the acquisition of a lowdose CT scan (26 mAs, $120 \mathrm{kV}, 0.5 \mathrm{~s}$ per rotation, 5-mm slice thickness) from the skull base to the mid-thigh or to the toes, depending on the location of the sarcoma. CT data were used for attenuation correction, characterization, and adequate anatomic localization of PET lesions. The PET scan was performed directly after CT data acquisition.

All patients fasted for at least $4 \mathrm{~h}$ before the injection of $0.22 \mathrm{mCi} / \mathrm{kg}$ body weight of FDG. Blood glucose levels did not exceed $150 \mathrm{mg} / \mathrm{dL}$. Scanning started 6090 min after the injection of the tracer. Patients sat quietly in a dimly lit room during the uptake phase. Patients were asked to void just prior to image acquisition. The CT and PET scans were obtained with the patient in a quiet respiration. Patients were allowed to drink water during the uptake period. They were instructed to avoid any kind of strenuous activity $24 \mathrm{~h}$ prior to the examination to avoid physiologic muscle FDG uptake. Intravenous contrast agent was administered in some patients.

Patients were examined in the supine position with an elevation of arms. CT scanning was started. The CT study took about 60-70 s. PET scans over the same 
Table 1 Distribution of the studied cases according to different parameters $(n=18)$

\begin{tabular}{|c|c|}
\hline & No. (\%) \\
\hline \multicolumn{2}{|l|}{ Sex } \\
\hline Male & $13(72.2 \%)$ \\
\hline Female & $5(27.8 \%)$ \\
\hline \multicolumn{2}{|l|}{ Age (years) } \\
\hline$<15$ & $9(50.0 \%)$ \\
\hline$\geq 15$ & $9(50.0 \%)$ \\
\hline Median (Min.-Max.) & $15.0(1.0-18.0)$ \\
\hline Mean \pm SD & $13.33 \pm 5.10$ \\
\hline \multicolumn{2}{|l|}{ 1ry site } \\
\hline Femur & $3(16.7 \%)$ \\
\hline Head and neck & $3(16.7 \%)$ \\
\hline Humerus & $2(11.1 \%)$ \\
\hline Fibula & $2(11.1 \%)$ \\
\hline Urinary bladder & $2(11.1 \%)$ \\
\hline Iliac bone & $2(11.1 \%)$ \\
\hline Tibia & $2(11.1 \%)$ \\
\hline Scapula & $1(5.6 \%)$ \\
\hline Abdominal wall & $1(5.6 \%)$ \\
\hline \multicolumn{2}{|l|}{ Histopathology } \\
\hline Osteosarcoma & 7 (38.9\%) \\
\hline Ewing sarcoma & $5(27.8 \%)$ \\
\hline Rhabdomyosarcoma & $4(22.2 \%)$ \\
\hline Myeloid sarcoma & $1(5.6 \%)$ \\
\hline Synovial sarcoma & $1(5.6 \%)$ \\
\hline \multicolumn{2}{|l|}{ SUV1 } \\
\hline Median (Min.-Max.) & $6.0(2.80-13.0)$ \\
\hline Mean $\pm S D$ & $6.71 \pm 3.22$ \\
\hline \multicolumn{2}{|l|}{ SUV2 } \\
\hline Median (Min.-Max.) & $2.30(1.20-20.0)$ \\
\hline Mean \pm SD & $4.86 \pm 5.80$ \\
\hline \multicolumn{2}{|l|}{ Histologic response } \\
\hline Good responder & $12(66.7 \%)$ \\
\hline Poor responder & $6(33.3 \%)$ \\
\hline
\end{tabular}

region were performed immediately after acquisition of the CT images (2-3 min/bed position).

The CT data were used for the attenuation correction of the PET emission data. PET/CT, PET, and CT images were displayed and reconstructed in the axial, sagittal, and coronal planes.

Side-by-side image interpretation was done by two experienced nuclear medicine physician and radiology physician.

Standard uptake values before (SUV1) and after (SUV2) chemotherapy were analyzed. SUV2 values were correlated with chemotherapeutic response assessed by histopathology which was performed to all patients following surgical excision of the tumors.

Statistical analysis of the data was fed to the computer and analyzed using IBM SPSS software package version 20.0. (Armonk, NY: IBM Corp). The Kolmogorov-Smirnov test was used to verify the normality of the distribution of variables. Mann-Whitney test was used to compare between two groups for not normally distributed quantitative variables. Agreement between markers was done using sensitivity, specificity, PPV, and NPV. Significance of the obtained results was judged at the $5 \%$ level.

\section{Results}

A total of $36 \mathrm{PET} / \mathrm{CT}$ studies were performed in 18 patients of our study: 13 patients were males (72.2\%) and 5 patients were females $(27.8 \%)$. Their ages ranged between 1 and 18 years with a mean age of 13.33 years (Table 1).

The most common primary tumor site was the femur, 3 cases (16.7\%). The most common histopathological diagnosis was osteosarcoma in 7 cases (38.9\%). These results were illustrated in (Table 1 ).

All patients underwent sequential 18F-FDG PET/CT before (PET/CT1) and after (PET/CT2) neoadjuvant chemotherapy. Maximum standardized uptake value (SUVmax) was measured in the primary lesion on PET/ CT1 (SUV1) and PET/CT2 (SUV2). SUV1 calculation of the 1ry tumor site ranged from 2.80-13.0 with a mean

Table 2 Relation between histologic response according to SUV

\begin{tabular}{|c|c|c|c|}
\hline & \multicolumn{2}{|l|}{ Histologic response } & \multirow[t]{2}{*}{$P$} \\
\hline & Poor responder $(n=6)$ & Good responder $(n=12)$ & \\
\hline \multicolumn{4}{|l|}{$\overline{\text { SUV1 }}$} \\
\hline Median (Min.-Max.) & $8.5(3.5-13.0)$ & $5.5(2.8-12.4)$ & \multirow[t]{2}{*}{0.151} \\
\hline Mean \pm SD & $8.5 \pm 3.7$ & $5.8 \pm 2.7$ & \\
\hline \multicolumn{4}{|l|}{ SUV2 } \\
\hline Median (Min.-Max.) & $8.1(3.6-20.0)$ & $1.8(1.2-3.2)$ & \multirow[t]{2}{*}{$<0.001$} \\
\hline Mean \pm SD & $10.7 \pm 7.2$ & $1.9 \pm 0.6$ & \\
\hline
\end{tabular}


Table 3 Agreement (sensitivity, specificity) for SUV2

\begin{tabular}{lllllllll}
\hline & Good responder $(n=12)$ & Poor responder $(n=6)$ & Sensitivity & Specificity & PPV & NPV & Accuracy \\
\hline SUV2 & $<2.5$ & 11 & 0 & 100.0 & 91.67 & 85.71 & 100.0 & 94.44 \\
& $\geq 2.5$ & 1 & 6 & & & & &
\end{tabular}

value of $6.71 \pm 3.22$. SUV2 calculation of the 1ry tumor site ranged from $1.20-20.0$ with a mean value of $4.86 \pm$ 5.80 (Table 1).

After the histopathological assessment, 12 patients (66.7\%) were good responders and 6 patients (33.3\%) were poor responders. SUV2 in good responders ranged from 1.2-3.2 with a mean value of $1.9 \pm 0.6$. Three patients in our study showed postchemotherapy increased SUV, all of which were poor responders. SUV2 in poor responders ranged from 3.6-20.0 with a mean value of $10.7 \pm 7.2$ (Table 2). SUV2 significantly correlated with the histologic response $(P<0.001)$, and the calculated sensitivity, specificity, PPV, NVP, and accuracy were 100.0, 91.67, 85.71, 100.0, and 94.44, respectively (Table 3).

Among the responders, 11 cases (91.6\%) showed no postchemotherapeutic morphological changes and only 1 case $(8.33 \%)$ showed postchemotherapeutic morphological changes in the form of moderate size reduction. Whereas among the nonresponders, 4 cases $(66.6 \%)$ showed size progression and 2 cases (33.3\%) showed no postchemotherapeutic morphologic changes (Figs. 1, 2, 3, 4, 5, 6 and 7).

\section{Discussion}

It was established that neoadjuvant and adjuvant chemotherapy increased survival rates in sarcoma patients [19]. Many trials were done to predict response to neoadjuvant therapy by diagnostic imaging to gain prognostic information or even to direct preoperative or operative therapy [20]. Therapy response in clinical practice is commonly assessed on the basis of change in the longest tumor diameter for soft-tissue sarcomas (response evaluation criteria in solid tumors); however, a reduction in the soft-tissue component of bone sarcomas in response

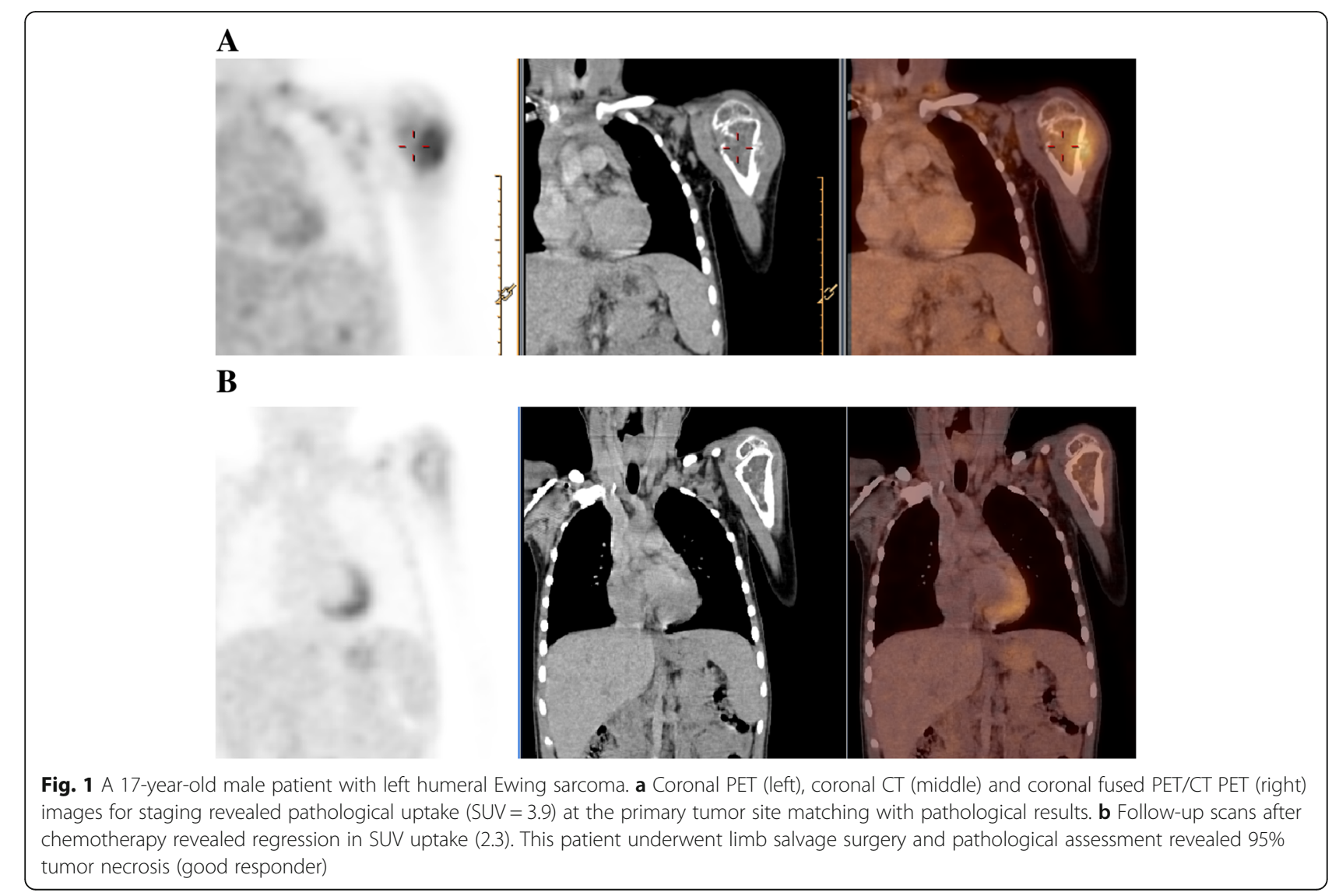


A

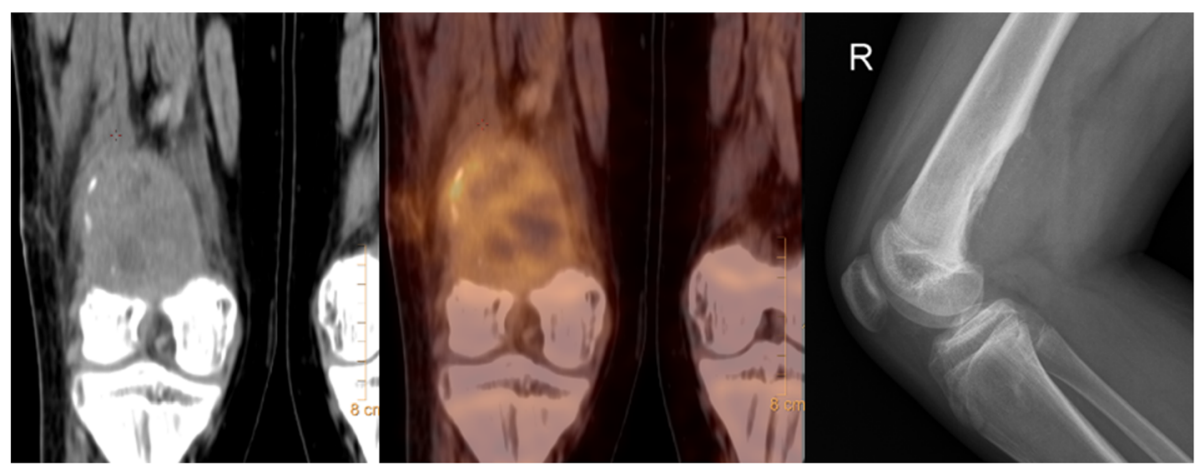

B

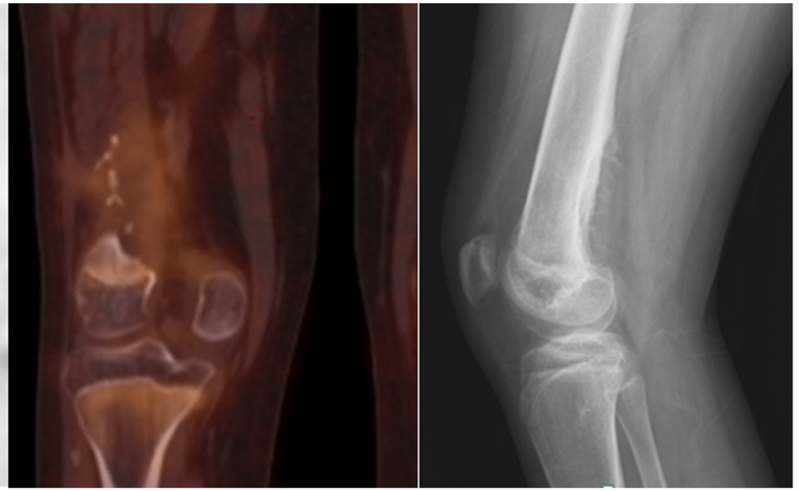

Fig. 2 A 17-year-old male with right femoral Ewing sarcoma. a Prechemotherapy coronal CT (left), PET/CT (middle) showed right femoral metabolically active FDG avid lesion with SUV about 4.7. X-ray (right) revealed posterior distal femoral lesion with a wide zone of transition and aggressive periosteal reaction. b Postchemotherapy coronal PET (left), PET/CT (middle) revealed metabolic regression regarding the distal femoral mass lesion (SUV 2.3). The patient underwent limb salvage surgery and pathological assessment revealed $95 \%$ tissue necrosis (good responder). No morphologic changes regarding conventional imaging features (right)

to chemotherapy does not necessarily correlate with favorable response [21].

The standard therapy for sarcoma is a combination of surgery and chemotherapy [22]. F-18 FDG PET examinations before and after neoadjuvant chemotherapy are commonly used now to monitor treatment response and to help time surgical treatment [23]. In addition, surgeons might decide on the type of operation depending on responsiveness to chemotherapy, as limb salvage cannot be recommended to patients in whom wide margins cannot be safely achieved [23]. Chemotherapy-induced necrosis is the most important prognostic factor in surgically managed patients [24].

18FFDG-PET/CT has a rising role to provide complementary information in sarcoma diagnosis and treatment planning and has been established as a diagnosing imaging tool in the staging, restaging, and assessment of the therapeutic response of soft-tissue as well as bone sarcomas [25].

It was difficult to collect extensive PET/CT data of pediatric malignancies owing to the relatively low incidence and wide range of pediatric malignancies as well as the relatively conservative application of PET/ CT in pediatric age. Thus, most of the previously published studies have focused on childhood lymphomas [26].

The main limitation in this study was the relatively small sample size of the patients and the heterogeneity of the sample as regards the histological diagnosis of different soft tissue and osseous sarcomas; however, the presence of sufficient radiological and pathological data for all the patients allowed adequate statistical analysis and interpretation of the results. The current study is one of the first to investigate the accuracy and added the diagnostic value of integrated 18F-FDG $\mathrm{PET} / \mathrm{CT}$ in the assessment of treatment response in pediatric sarcomas. Previous studies mainly assessed the performance of molecular imaging for initial tumor staging, and they did not focus on cancer subtypes like Ewing sarcoma [27]. The results in the current study indicated that integrated 18F-FDG PET/ $\mathrm{CT}$ is an effective noninvasive diagnostic tool for assessment of therapy response in pediatric patients who have a history of bone or soft tissue sarcoma. 
A
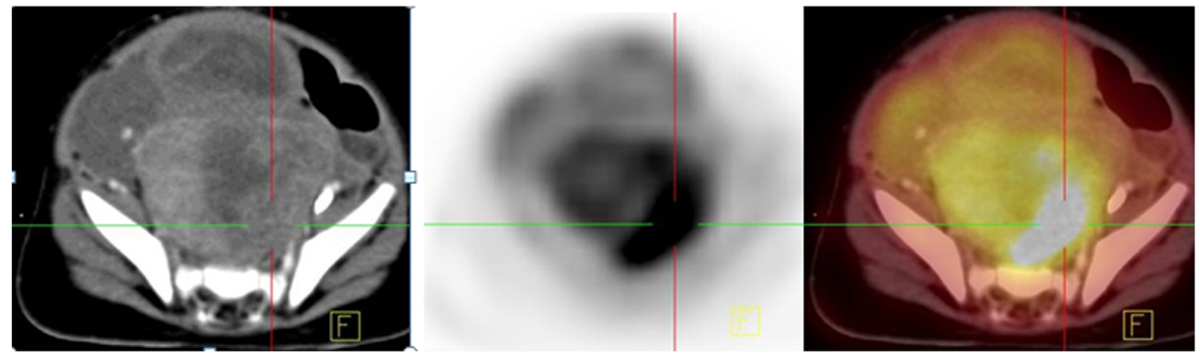

B
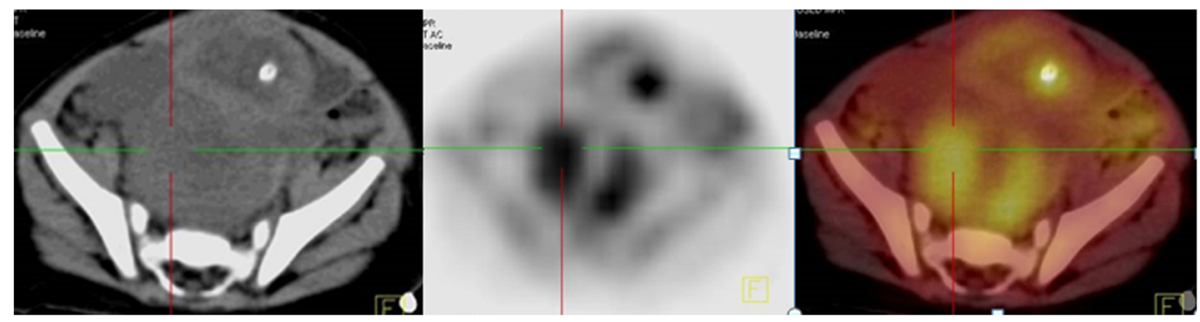

Fig. 3 A 1-year-old boy presented with bladder mass which proved to be embryonal rhabdomyosarcoma came for assessment of treatment response. a Prechemotherapy axial CT (left), axial PET (middle), and axial fused PET/CT (right) revealed metabolically active FDG avid primary mass (with SUV = 5.4). b Postchemotherapy axial CT (left), axial PET (middle), and axial fused PET/CT (right) revealed regression of the FDG uptake (SUV = 3.6). Radical cystectomy was done and the pathology showed that there was $85 \%$ viable tumor (poor responder)

Good responders to chemotherapy considered to have $90 \%$ or greater necrosis and poor responders who had less than $90 \%$ necrosis. This is in concordance with what Hawkins et al. mentioned [28].

SUV2 of 2.5 is considered a cutoff value between pathological and nonpathological uptake; this is also in agreement with Andrei Iagaru et al. [23].

Hawkins et al. [28] stated that using a cutoff SUVmax of less than 2.8, 18FFDG PET/CT differentiated responders from nonresponders with up to $100 \%$ accuracy after completion of neoadjuvant therapy.

Kumar et al. [29] also considered SUV2 as an accurate noninvasive prognostic indicator; they found that disease progression-free survival (PFS) is $72 \%$ for SUV2 less than 2.5 versus $27 \%$ for SUV2 more than or equal to 2.5 .

Rosen et al. [30], Picci et al. [31], and Asha Kandathil et al. [1] concluded that histologic response, measured
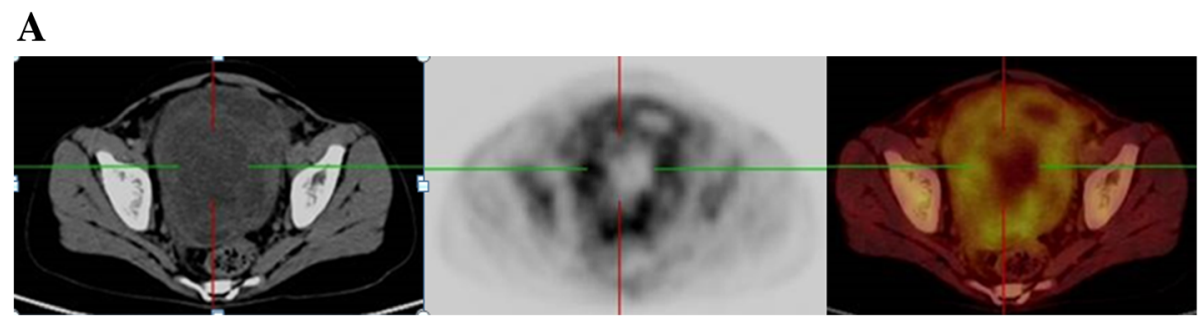

B

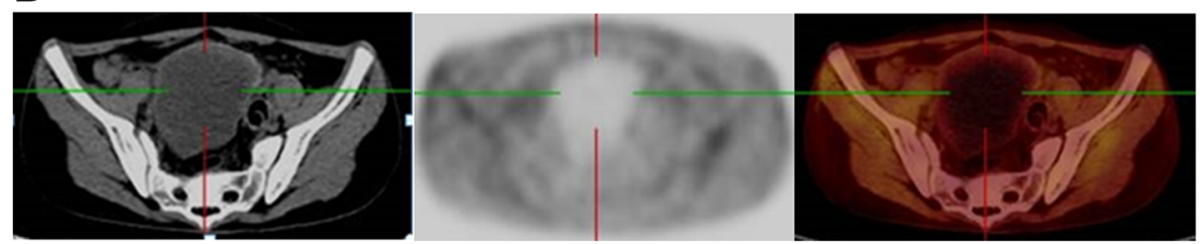

Fig. 4 A 13-year-old boy presented with urinary bladder mass; the mass was proven by a biopsy to be embryonal rhabdomyosarcoma. a Prechemotherapy axial CT (left), axial PET (middle), and axial fused PET/CT (right) revealed metabolically active FDG avid 1ry tumor lesion (SUV = 2.9). b Postchemotherapy axial CT (left), axial PET (middle), and axial fused PET/CT (right) revealed marked regression of the FDG uptake (SUV= 1.5) as well as moderate size regression. The mass was excised without tumor viability detected on histopathology 


\section{A}

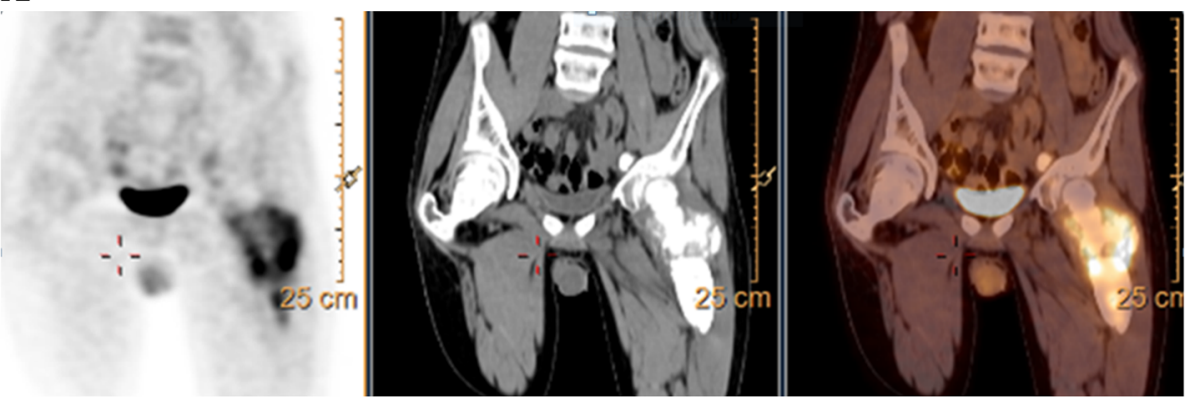

B

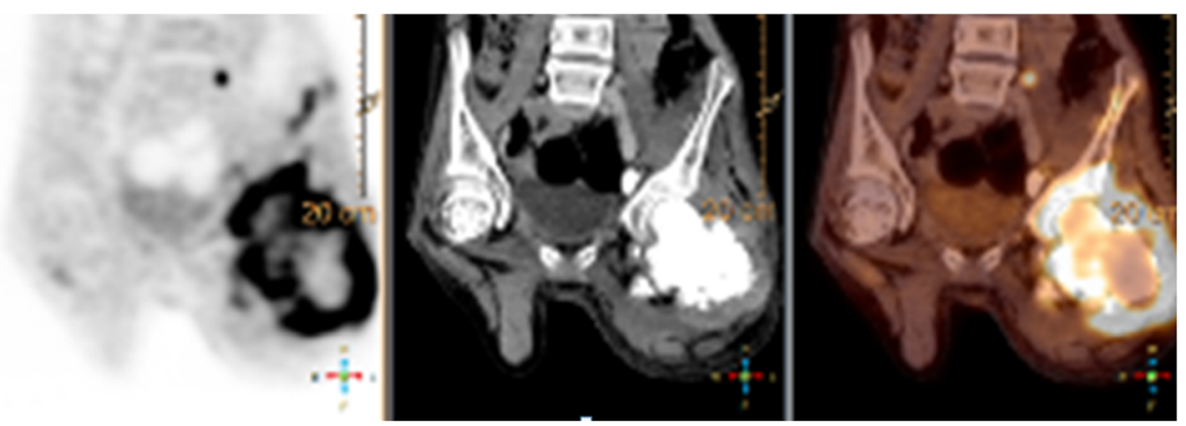

Fig. 5 A 18-year-old male with left femoral osteosarcoma. a Prechemotherapy coronal PET (left), coronal CT (middle), and coronal fused PET/CT (right) images revealed metabolically active FDG avid 1rt tumor (SUV 9). b Prechemotherapy coronal PET (left), coronal CT (middle), and coronal fused PET/CT (right) images revealed progression regarding size and FDG acidity (SUV = 19). Amputation was done and histopathology showed that there was $90 \%$ viable tumor (poor responder)

$\mathbf{A}$
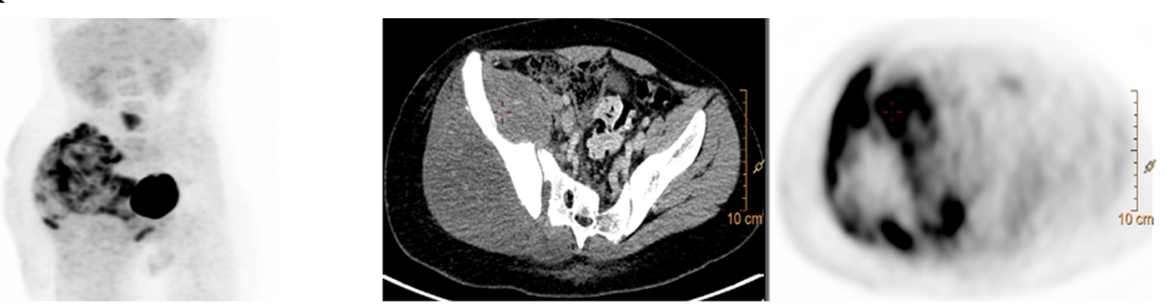

B
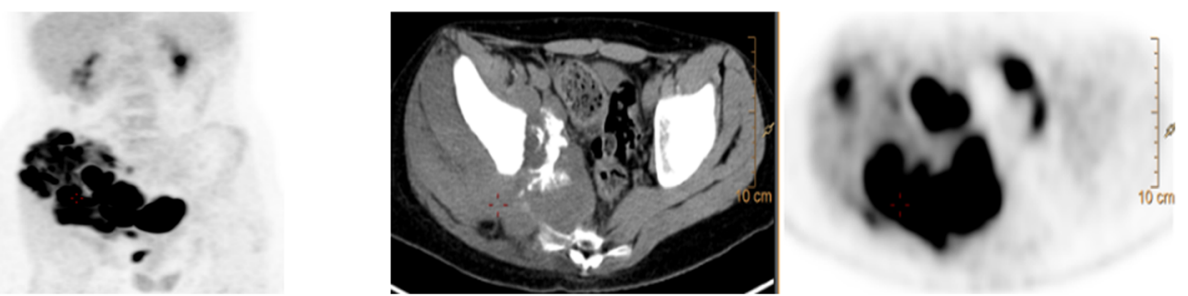

Fig. 6 A 16-year-old male with right iliac bone osteosarcoma. a Prechemotherapy coronal PET, axial CT, and axial PET revealed high pathological FDG uptake (SUV=12) at the primary tumor site. Postchemotherapy coronal PET, axial $C T$, and axial PET revealed disease progression regarding size and FDG avidity (20 in the study). Amputation was done and histopathology showed that there was $90 \%$ viable tumor (poor responder) 
A
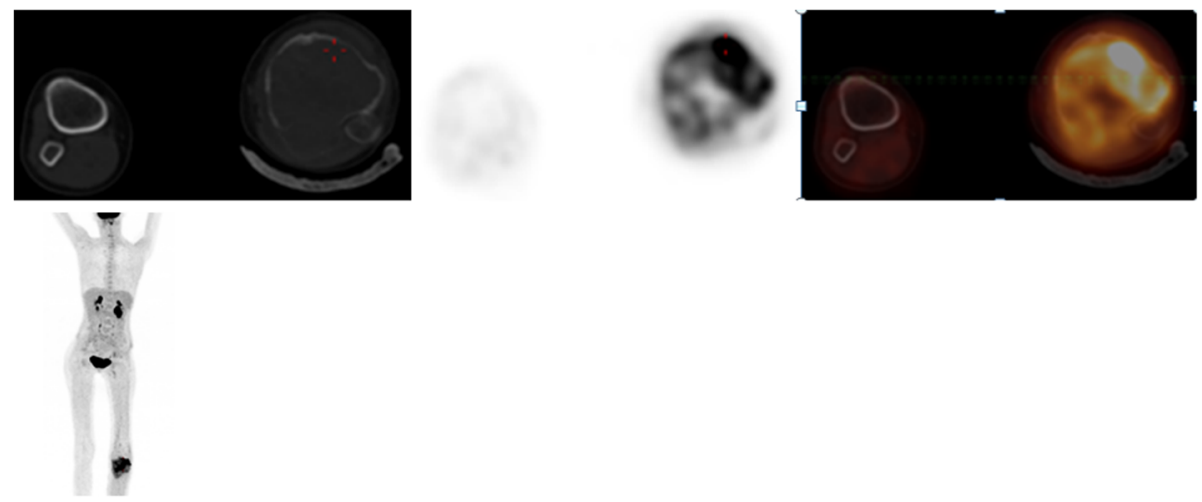

B
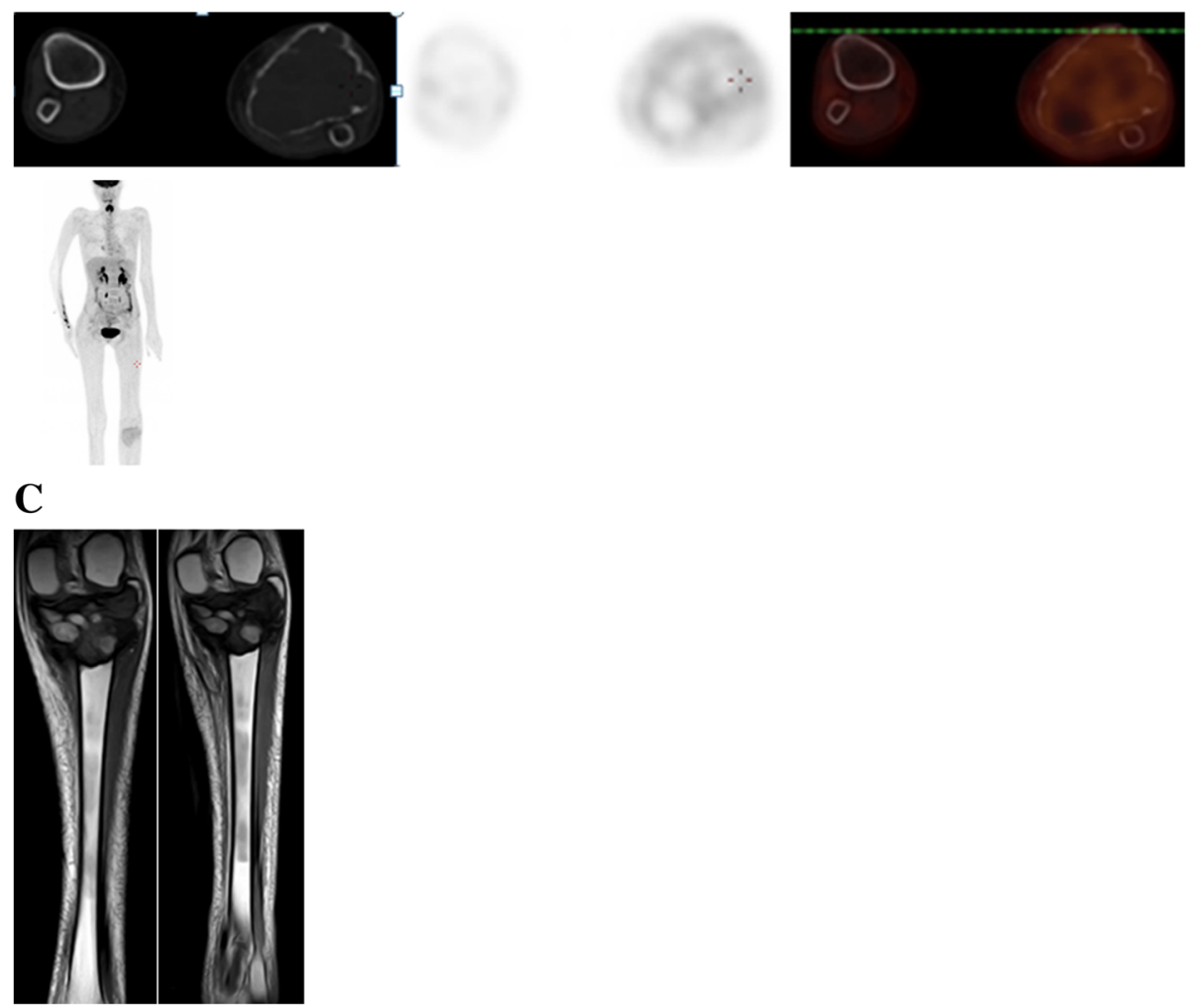

Fig. 7 A 14-year-old female patient suffered from left proximal tibia telangiectatic osteosarcoma. a Prechemotherapy PET/CT revealed metabolically active FDG left proximal tibial mass lesion (SUV $=12.4)$. b After the end of treatment, PET/CT revealed no pathological uptake $(S U V=1.8)$. The patient underwent limb salvage surgery and surgical pathology revealed good histological response (100\% tissue necrosis). c No corresponding morphologic improvement was seen between the premanagement MRI (left) and postmanagement one (right)

by the percentage of necrotic tumor cells remaining after neoadjuvant chemotherapy, has prognostic value in predicting event-free survival (EFS) in children with osteosarcoma (OS) and Ewing sarcoma.

SUV2 was found to significantly correlated with the histologic response $(P<0.001)$; the calculated sensitivity, specificity, PPV, NVP, and accuracy were 100.0, 91.67, $85.71,100.0$, and 94.44, respectively. This is in concordance with Douglas et al. [28] who proved that SUV2 was correlated with a histologic response in pediatric bone sarcomas.

The change in SUV after chemotherapy was found not matching with morphological changes detected on CT images. Among the responders, only 1 case (8.33\%) showed postchemotherapy morphologic changes in the form of moderate size reduction (Fig. 4), and the other 11 cases $(91.6 \%)$ showed no postchemotherapy morphologic changes. 
Murphy et al. [32] and McCarville et al. [33] stated that sarcomas, frequently do not change in size in response to chemotherapy, radiographic evaluation of response by computed tomography (CT), or magnetic resonance imaging (MRI), do not distinguish between responders and nonresponders and that only minimal morphologic change may be detected at conventional imaging in spite of a significant reduction in tumor viability after therapy.

Denecke et al. [21] and Bredella et al. [34] reported that an overall tumor SUV and SUVmax on posttreatment 18F-FDG PET/CT scans were more accurate for the assessment of treatment response than changes in tumor size.

\section{Conclusions}

18F-FDG PET/CT provides a reliable noninvasive diagnostic tool in the assessment of response to preoperative chemotherapy in pediatric sarcoma which is valuable in the decision of the type of surgery specially in cases with limb involvement whether considering limb salvage technique or amputation and also of benefit in assessment of prognosis of the patients following treatment.

\section{Abbreviations}

CMR: Complete metabolic response; CT: Computed tomography; DFS: Disease-free survival; EFS: Event-free survival; FDG PET/CT: 18Ffluorodeoxyglucose positron emission tomography/computed tomography; PFS: Progression-free survival; STS: Soft-tissue sarcoma; SUVmax: Maximum standardized uptake value

\section{Acknowledgements}

Not applicable

\section{Authors' contributions}

AR shared in obtaining the radiological data in this study, shared in the interpretation of results of the study, shared in the design and coordination of the study, and helped in drafting the manuscript. AA shared in the interpretation of the results of this study, performed the statistical analysis, shared in the design and coordination of the study, and helped in drafting the manuscript. SF shared in obtaining the clinical data included in this study. WO shared in obtaining the radiological data in this study and shared in the interpretation of the results of the study. KM shared in the final revision of the manuscript of this study and revised the obtained results and data. All authors read and approved the final manuscript.

\section{Funding}

Not applicable

\section{Availability of data and materials}

The datasets used and/or analyzed during the current study are available from the corresponding author on reasonable request.

\section{Ethics approval and consent to participate}

Approval for this study was obtained from the Research Ethics Committee of Alexandria Faculty of Medicine. All study procedures were carried out in accordance with the Declaration of Helsinki regarding research involving human subjects.

\section{Consent for publication}

All patients included in this research gave written informed consent to publish the data contained within this study. If the patient was less than 16 years old, deceased, or unconscious when consent for publication was requested, written informed consent for the publication of this data was given by their parent or legal guardian.

\section{Competing interests}

The authors declare that they have no competing interests.

\section{Author details}

${ }^{1}$ Diagnostic and Interventional Radiology Department, Faculty of Medicine, Alexandria University, Alexandria, Egypt. ${ }^{2}$ Oncology Department, Faculty of Medicine, Alexandria University, Alexandria, Egypt. ${ }^{3}$ Nuclear Medicine and Molecular Imaging Department, National cancer institute, Cairo University, Cairo, Egypt.

Received: 15 June 2019 Accepted: 26 July 2019

Published online: 07 September 2019

\section{References}

1. Kandathil A, Subramaniam RM (2017) PET/computed tomography and precision medicine: musculoskeletal sarcoma. PET Clin 12:475-488

2. Savina M, Le Cesne A, Blay JY, Ray-Coquard I, Mir O, Toulmonde M et al (2017) Patterns of care and outcomes of patients with METAstatic soft tissue SARComa in a real-life setting: the METASARC observational study. BMC Med. 15:78

3. Quartuccio N, Fox J, Kuk D, Wexler LH, Baldari S, Cistaro A et al (2015) Pediatric bone sarcoma: diagnostic performance of (1)(8)F-FDG PET/CT versus conventional imaging for initial staging and follow-up. AJR Am J Roentgenol. 204:153-160

4. Bakhshi S, Radhakrishnan V (2010) Prognostic markers in osteosarcoma. Expert Rev Anticancer Ther. 10:271-287

5. Treglia G, Salsano M, Stefanelli A, Mattoli MV, Giordano A, Bonomo L (2012) Diagnostic accuracy of (1)(8)F-FDG-PET and PET/CT in patients with Ewing sarcoma family tumours: a systematic review and a meta-analysis. Skeletal Radiol. 41:249-256

6. Tinkle CL, Weinberg V, Braunstein SE, Wustrack R, Horvai A, Jahan T et al (2015) Intraoperative radiotherapy in the management of locally recurrent extremity soft tissue sarcoma. Sarcoma. 2015:913565

7. Choi YY, Kim JY, Yang SO (2014) PET/CT in benign and malignant musculoskeletal tumors and tumor-like conditions. Semin Musculoskelet Radiol. 18:133-148

8. Benz MR, Czernin J, Allen-Auerbach MS, Tap WD, Dry SM, Elashoff D et al (2009) FDG-PET/CT imaging predicts histopathologic treatment responses after the initial cycle of neoadjuvant chemotherapy in high-grade soft-tissue sarcomas. Clin Cancer Res. 15:2856-2863

9. Quak E, van de Luijtgaarden AC, de Geus-Oei LF, van der Graaf WT, Oyen W (2011) Clinical applications of positron emission tomography in sarcoma management. Expert Rev Anticancer Ther. 11:195-204

10. Evilevitch V, Weber WA, Tap WD, Allen-Auerbach M, Chow K, Nelson SD et al (2008) Reduction of glucose metabolic activity is more accurate than change in size at predicting histopathologic response to neoadjuvant therapy in high-grade soft-tissue sarcomas. Clin Cancer Res. 14:715-720

11. Picci P, Bohling T, Bacci G, Ferrari S, Sangiorgi L, Mercuri M et al (1997) Chemotherapy-induced tumor necrosis as a prognostic factor in localized Ewing's sarcoma of the extremities. J Clin Oncol. 15:1553-1559

12. Bielack SS, Kempf-Bielack B, Delling G, Exner GU, Flege S, Helmke K et al (2002) Prognostic factors in high-grade osteosarcoma of the extremities or trunk: an analysis of 1,702 patients treated on neoadjuvant cooperative osteosarcoma study group protocols. J Clin Oncol. 20:776-790

13. Costelloe CM, Macapinlac HA, Madewell JE, Fitzgerald NE, Mawlawi OR, Rohren EM et al (2009) 18F-FDG PET/CT as an indicator of progression-free and overall survival in osteosarcoma. J Nucl Med. 50:340-347

14. Hawkins DS, Conrad EU 3rd, Butrynski JE, Schuetze SM, Eary JF (2009) [F-18]fluorodeoxy-D-glucose-positron emission tomography response is associated with outcome for extremity osteosarcoma in children and young adults. Cancer. 115:3519-3525

15. Casey DL, Wexler LH, Fox JJ, Dharmarajan KV, Schoder H, Price AN et al (2014) Predicting outcome in patients with rhabdomyosarcoma: role of [(18)fffluorodeoxyglucose positron emission tomography. Int J Radiat Oncol Biol Phys. 90:1136-1142

16. Provisor AJ, Ettinger LJ, Nachman JB, Krailo MD, Makley JT, Yunis EJ et al (1997) Treatment of nonmetastatic osteosarcoma of the extremity with preoperative and postoperative chemotherapy: a report from the Children's Cancer Group. J Clin Oncol. 15:76-84 
17. Sheikhbahaei S, Marcus C, Hafezi-Nejad N, Taghipour M, Subramaniam RM (2015) Value of FDG PET/CT in patient management and outcome of skeletal and soft tissue sarcomas. PET Clin. 10:375-393

18. Eary JF, Conrad EU, O'Sullivan J, Hawkins DS, Schuetze SM, O'Sullivan F (2014) Sarcoma mid-therapy [F-18]fluorodeoxyglucose positron emission tomography (FDG PET) and patient outcome. J Bone Joint Surg Am. 96: 152-158

19. Arndt CA, Rose PS, Folpe AL, Laack NN (2012) Common musculoskeletal tumors of childhood and adolescence. Mayo Clin Proc. 87:475-487

20. Qin Z, Tang Y, Wang H, Cai W, Fu H, Li J et al (2015) Use of 18F-FDG-PET-CT for assessment of response to neoadjuvant chemotherapy in children with Wilms tumor. J Pediatr Hematol Oncol. 37:396-401

21. Denecke T, Hundsdorfer P, Misch D, Steffen IG, Schonberger S, Furth C et al (2010) Assessment of histological response of paediatric bone sarcomas using FDG PET in comparison to morphological volume measurement and standardized MRI parameters. Eur J Nucl Med Mol Imaging. 37:1842-1853

22. Angelini A, Ceci F, Castellucci P, Graziani T, Polverari G, Trovarelli G et al (2017) The role of (18)F-FDG PET/CT in the detection of osteosarcoma recurrence. Eur J Nucl Med Mol Imaging. 44:1712-1720

23. lagaru A, Masamed R, Chawla SP, Menendez LR, Fedenko A, Conti PS (2008) F-18 FDG PET and PET/CT evaluation of response to chemotherapy in bone and soft tissue sarcomas. Clin Nucl Med. 33:8-13

24. Eilber FC, Rosen G, Eckardt J, Forscher C, Nelson SD, Selch M et al (2001) Treatment-induced pathologic necrosis: a predictor of local recurrence and survival in patients receiving neoadjuvant therapy for high-grade extremity soft tissue sarcomas. J Clin Oncol. 19:3203-3209

25. Bastiaannet E, Groen H, Jager PL, Cobben DC, van der Graaf WT, Vaalburg W et al (2004) The value of FDG-PET in the detection, grading and response to therapy of soft tissue and bone sarcomas: a systematic review and metaanalysis. Cancer Treat Rev. 30:83-101

26. Schaefer NG, Taverna C, Strobel K, Wastl C, Kurrer M, Hany TF (2007) Hodgkin disease: diagnostic value of FDG PET/CT after first-line therapy-is biopsy of FDG-avid lesions still needed? Radiology. 244:257-262

27. Charest M, Hickeson M, Lisbona R, Novales-Diaz JA, Derbekyan V, Turcotte RE (2009) FDG PET/CT imaging in primary osseous and soft tissue sarcomas: a retrospective review of 212 cases. Eur J Nucl Med Mol Imaging. 36:1944-1951

28. Hawkins DS, Rajendran JG, Conrad EU 3rd, Bruckner JD, Eary JF (2002) Evaluation of chemotherapy response in pediatric bone sarcomas by [F-18]fluorodeoxy-D-glucose positron emission tomography. Cancer. 94:3277-3284

29. Kumar R, Chauhan A, Vellimana AK, Chawla M (2006) Role of PET/PET-CT in the management of sarcomas. Expert Rev Anticancer Ther 6:1241-1250

30. Rosen G, Caparros B, Groshen S, Nirenberg A, Cacavio A, Marcove RC et al (1984) Primary osteogenic sarcoma of the femur: a model for the use of preoperative chemotherapy in high risk malignant tumors. Cancer Invest. 2: 181-192

31. Picci P, Rougraff BT, Bacci G, Neff JR, Sangiorgi L, Cazzola A et al (1993) Prognostic significance of histopathologic response to chemotherapy in nonmetastatic Ewing's sarcoma of the extremities. J Clin Oncol. 11:1763-1769

32. Murphy WA Jr (1991) Imaging bone tumors in the 1990s. Cancer. 67:1169-1176

33. McCarville MB, Christie R, Daw NC, Spunt SL, SCJAJoR K (2005) PET/CT in the evaluation of childhood sarcomas. AJR Am J Roentgenol 184:1293-1304

34. Bredella MA, Caputo GR, Steinbach LS (2002) Value of FDG positron emission tomography in conjunction with MR imaging for evaluating therapy response in patients with musculoskeletal sarcomas. AJR Am J Roentgenol. 179:1145-1150

\section{Publisher's Note}

Springer Nature remains neutral with regard to jurisdictional claims in published maps and institutional affiliations.

\section{Submit your manuscript to a SpringerOpen ${ }^{\circ}$ journal and benefit from:}

- Convenient online submission

- Rigorous peer review

- Open access: articles freely available online

- High visibility within the field

- Retaining the copyright to your article

Submit your next manuscript at $\boldsymbol{\nabla}$ springeropen.com 